Fetal Diagnosis and Therapy

\title{
Leukocyte Counts and Other Hematological Values in Twin-Twin Transfusion Syndrome and Twin Anemia-Polycythemia Sequence
}

\author{
G. Laurien Visser ${ }^{\mathrm{a}}$ Lisanne S.A. Tollenaar ${ }^{\mathrm{b}}$ Vincent Bekker ${ }^{\mathrm{a}} \quad$ Arjan B. te Pas $^{\mathrm{a}}$ \\ Arjan C. Lankester ${ }^{c}$ Dick Oepkes $^{b}$ Enrico Lopriore ${ }^{a}$ Lianne Verbeek $^{a}$ \\ aDivision of Neonatology, Department of Pediatrics, Leiden University Medical Center, Leiden, The Netherlands; \\ ${ }^{b}$ Department of Obstetrics, Leiden University Medical Center, Leiden, The Netherlands; ' Division of Immunology, \\ Hematology and Stem Cell Transplantation, Department of Pediatrics, Leiden University Medical Center, Leiden, \\ The Netherlands
}

\section{Keywords}

Monochorionic twins · Twin-twin transfusion syndrome .

Twin anemia-polycythemia sequence $\cdot$ Leukopenia .

Leukocyte count

\begin{abstract}
Objective: The aim of this study was to evaluate the differences in leukocyte counts at birth between donors and recipients with twin-twin transfusion syndrome (TTTS) or twin anemia-polycythemia sequence (TAPS). Methods: We performed a retrospective cohort study in monochorionic twin pairs with TTTS or TAPS. TTS and TAPS cases treated with fetoscopic laser surgery were excluded. Primary outcome was the difference in leukocyte levels at birth between donor and recipient twins and the presence of leukopenia (defined as leukocyte count $\left.<4 \times 10^{9} / \mathrm{L}\right)$. Secondary outcomes included early-onset sepsis, necrotizing enterocolitis, use of antibiotics during admission, and neonatal mortality. Results: We included 99 twins pairs, of which 61 twin pairs were affected by TAPS and 38 twin pairs by TTTS. The mean leukocyte count at birth in donors and recipients was $7.5 \times 10^{9} / \mathrm{L}$ versus $7.4 \times 10^{9} / \mathrm{L}(p=0.936)$, respectively. Leukopenia was significantly more common in donor twins compared to re-
\end{abstract}

cipient twins (7.1\% [7/99] vs. $0 \%$ [0/99], $p=0.016)$. Of the 7 donors with leukopenia, 6 were affected by TAPS and 1 by TTTS. Overall, donors were more often affected by early-onset sepsis than recipients, $23.7 \%$ (23/97) versus 13\% (13.7/95) $(p=0.049)$, respectively. Conclusions: Leukocyte counts at birth in twins with TTTS or TAPS are similar between donors and recipients, but TAPS donors are at an increased risk of leukopenia. Overall, TTTS and TAPS donors seem to be at an increased risk of early-onset neonatal sepsis compared to recipient twins.

(c) 2019 The Author(s) Published by S. Karger AG, Basel

\section{Introduction}

Vascular anastomoses are almost invariably present in all monochorionic twin placentas and may lead to several complications including twin-twin transfusion syndrome (TTTS) and twin anemia-polycythemia sequence (TAPS). TTTS occurs in $10 \%$ of the monochorionic twins and is characterized by the development of twin oligo-polyhydramnios sequence. In contrast, TAPS is characterized by large inter-twin hemoglobin $(\mathrm{Hb})$ differences without signs 
of twin oligo-polyhydramnios sequence. TAPS can be detected in $2-13 \%$ of the TTTS pregnancies after incomplete fetoscopic laser surgery (post-laser TAPS) and can also occur spontaneously in about $3-5 \%$ of the monochorionic twin pregnancies (spontaneous TAPS) [1]. TAPS cases are characterized by the presence of only few minuscule arteriovenous placental anastomoses allowing slow and chronic inter-twin blood transfusion, resulting in chronic anemia in donors and chronic polycythemia in recipients.

In TTTS and TAPS cases treated conservatively (without fetoscopic laser coagulation of the vascular anastomoses), $\mathrm{Hb}$ levels are significantly lower in donor twins due to inter-twin blood transfusion $[2,3]$. In addition, other inter-twin differences in blood measurements have been reported, including lower levels of albumin and total protein in donors, suggesting that inter-twin transfusion in TTTS and TAPS may not only be limited to transfusion of red blood cells $[2,4]$.

In one small study in 5 TTTS cases, transient neutropenia was reported in all 5 donor twins [5], suggesting that hematologic differences in TTTS and TAPS may also involve inter-twin transfusion of white blood cells. Leukopenia and especially neutropenia at birth are associated with adverse clinical conditions, including neonatal sepsis, necrotizing enterocolitis (NEC), and neonatal mortality [6].

However, additional information on differences in leukocyte levels in TTTS and TAPS is currently lacking. We therefore performed a large retrospective study to evaluate the differences in leukocyte counts and leukopenia at birth in donor twins and recipient twins with TTTS or TAPS.

\section{Methods}

All consecutive monochorionic twin pairs with TAPS or TTTS not treated with complete laser therapy and delivered at the Leiden University Medical Center between March 2002 and August 2018 were included in this study. The Leiden University Medical Center is a tertiary care center managing all types of complications of monochorionic pregnancies and a national referral center for fetoscopic laser treatment for TTTS and TAPS. We excluded all TTTS or TAPS twin pregnancies treated with successful (and thus complete) fetoscopic laser coagulation of the vascular anastomoses and twin pregnancies with single or double fetal demise. As a result, only twins with anastomoses in their placenta were included.

TAPS was diagnosed antenatally or postnatally. Antenatal diagnosis was based on an increased middle cerebral artery peak systolic velocity of $>1.5$ multiples of the median in the donor and a reduced middle cerebral artery peak systolic velocity of $<1.0$ multiples of the median in the recipient. Postnatally, TAPS was diagnosed in case of an inter-twin Hb difference $>8 \mathrm{~g} / \mathrm{dL}$ and at least one of the following: reticulocyte count ratio $>1.7$ or the presence of only small (diameter $<1 \mathrm{~mm}$ ) vascular anastomoses in the pla- centa, detected through color dye injection of the placenta [7]. TTTS was diagnosed using the Eurofoetus criteria, with a cut-off at the deepest vertical pocket of amniotic fluid in the donor at $\leq 2$ $\mathrm{cm}$ and in the recipient at $\geq 8 \mathrm{~cm}$ within the first 20 weeks of gestation, and at $\geq 10 \mathrm{~cm}$ after gestational week 20 [8].

At birth, levels of $\mathrm{Hb}$, leukocyte count and differentiation, and thrombocytes were measured routinely in all twins according to our local protocol. Blood samplings were primarily obtained from the umbilical cord. If umbilical cord blood was not available, venous blood samplings were obtained on day 1 . In case of suspicion of sepsis, C-reactive protein and blood culture were also obtained. Thrombocytopenia was defined as thrombocytes $<150 \times 10^{9} / \mathrm{L}$. Anemia was defined as the need for blood transfusion $<24 \mathrm{~h}$ after birth. Polycythemia was defined as a venous hematocrit level $\geq 65 \%$ and the need for partial exchange transfusion $<24 \mathrm{~h}$ after birth.

We recorded the following perinatal variables: gestational age at birth, birth weight, birth weight discordance, Quintero stage at diagnosis in TTTS twins, maternal antibiotic use and maternal risk factors for perinatal sepsis such as maternal fever, maternal Group B Streptococcus (GBS) carrier, former child with GBS sepsis, prolonged rupture of membranes, or meconium-stained fluid. Maternal fever was defined as a maternal temperature $>38.0^{\circ} \mathrm{C}$ during labor. Prolonged rupture of membranes was defined as rupture of membranes $>24 \mathrm{~h}$ prior to delivery. Birth weight discordance was calculated by dividing the difference in birth weight between twins by the birth weight of the larger twin.

The following neonatal data were collected: small for gestational age (SGA) defined as a birth weight $<$ p10, early-onset sepsis, antibiotic treatment during admission at our neonatal intensive care unit, NEC $\geq$ stage 2 (according to Bell's criteria [9]) and neonatal mortality. Diagnosis of early-onset sepsis was based on a positive blood culture within the first $72 \mathrm{~h}$ postpartum (proven sepsis) or the need for administration of a full course (5-7 days) of antibiotics due to risk factors or clinical signs of sepsis, in the absence of a positive blood culture (suspected sepsis). Neonatal mortality was defined as death $<28$ days after birth.

Primary outcome was the difference in leukocyte levels at birth and the incidence of leukopenia between donor and recipient twins. Leukopenia was defined as a leukocyte count $<4 \times 10^{9} / \mathrm{L}$. Secondary outcomes were the incidence of early-onset sepsis, NEC, antibiotic use, and neonatal mortality.

\section{Statistics}

Data are reported as medians and interquartile ranges. Results of continuous variables within twin pairs were analyzed using the related-samples Wilcoxon signed rank test. For analyses of paired nominal variables, the $\mathrm{McNemar}$ test was used. For statistical analyses, two-sided tests were used, and $p<0.05$ was considered statistically significant. Analysis was performed using SPSS version 23 (SPSS, Inc., Chicago, IL, USA).

\section{Results}

During the study period, 99 twin pairs fulfilled the inclusion criteria, of which 38 were TTTS twin pairs and 61 TAPS twin pairs. The baseline characteristics are summarized in Table 1. 
Table 1. Baseline characteristics in twins affected by TTTS and/or TAPS

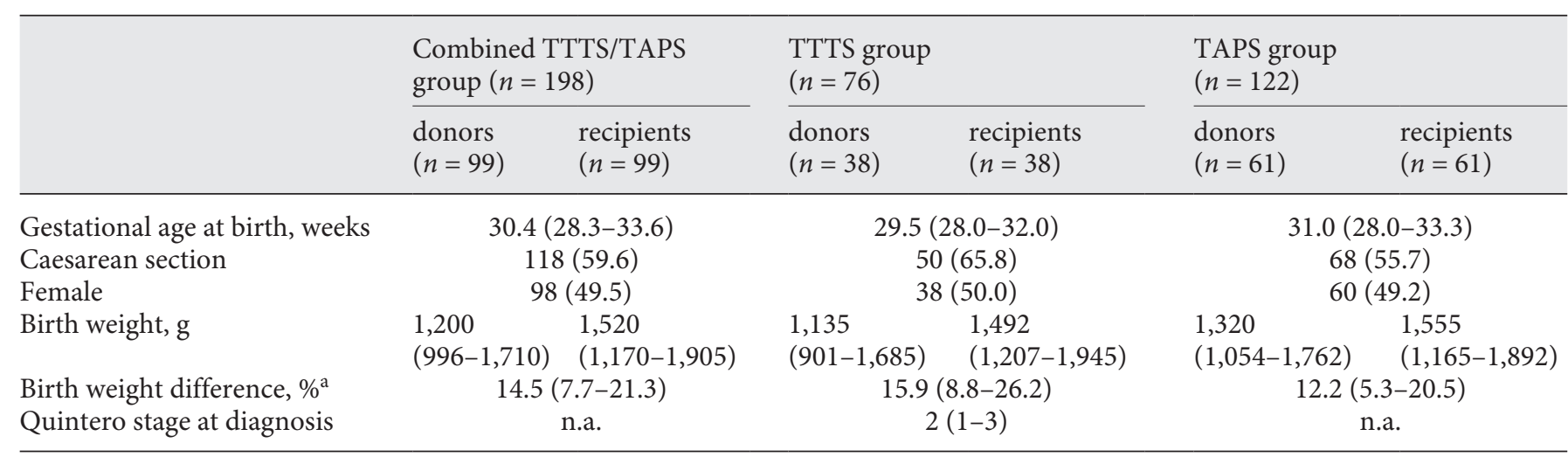

Values are given as $n(\%)$ or median (interquartile range). n.a., not applicable. ${ }^{a}$ Calculated as: ((birth weight of the larger twin - birth weight of the smaller twin) / (birth weight of the larger twin) $) \times 100$.

In the TAPS group, 70\% (43/61) were post-laser TAPS cases, and 30\% (18/61) were spontaneous TAPS twins. In total, $25.9 \%(15 / 58)$ received an intrauterine transfusion (with or without partial exchange transfusion), and 74.1\% were managed expectantly.

In the TTTS group, $47.4 \%(18 / 38)$ of the twin pairs had Quintero stage 1 at diagnosis, $13.2 \%$ (5/38) stage 2, 31.6\% $(12 / 38)$ stage 3 , and $7.9 \%$ (3/38) stage 4 . Of all TTTS twin pairs, $66 \%(25 / 38)$ were treated with serial amnioreduction, and $34 \%(13 / 38)$ were managed expectantly.

Results of the complete blood count in the TTTS and TAPS twins are shown in Table 2. The mean leukocyte levels were similar between donor and recipient twins, $7.5 \times$ $10^{9} / \mathrm{L}$ and $7.4 \times 10^{9} / \mathrm{L}(p=0.936)$, respectively. Post-hoc analysis showed that the incidence of SGA in the group of TAPS neonates with leukopenia was similar to that in the group of TAPS neonates without leukopenia, 50\% (3/6) and $40 \%(46 / 122)(p=0.616)$, respectively. Leukopenia (leukocyte count $\left.<4 \times 10^{9} / \mathrm{L}\right)$ in the combined TTTS/TAPS group was only seen in donors and not in any of the recipients (7.1\% [7/99] vs. $0 \%$ [0/99], $p=0.016)$. This difference was primarily due to an increased risk of leukopenia in TAPS donors compared to TAPS recipients $(9.8 \%$ [6/61] vs. $0 \%$ $[0 / 61], p=0.031$ ). Leukocyte differentiation was not different between donors and recipients in all groups (combined TTTS/TAPS group, TTTS group or TAPS group alone).

$\mathrm{Hb}$ levels were significantly lower in donor twins compared to recipient twins, 13.1 (11.4-14.9) g/dL versus 17.6 $(16.0-19.7) \mathrm{g} / \mathrm{dL}(p<0.001)$, respectively. Anemia was only seen in donor twins and not in any of the recipients of both groups. Recipients were significantly more often affected by polycythemia compared to donors $(22.9 \%$ vs. $0 \%, p=$
0.008 , in TTTS twins and $46.3 \%$ vs. $0 \%, p<0.001$, in TAPS twins). In TAPS twins, reticulocyte count was significantly higher in donor twins compared to recipient twins, 125 versus $43 \%$ o $(p<0.001)$, respectively. In TTTS twins, no significant difference in reticulocyte count between donors and recipients was found. In the TTTS group, donor twins had significantly lower thrombocyte levels and were more often affected by thrombocytopenia compared to recipient twins, $186(120-220) \times 10^{9} / \mathrm{L}$ versus $202(163-238) \times 10^{9} / \mathrm{L}$ $(p=0.030)$ and $35.1 \%$ versus $11.1 \%(p=0.004)$. In the TAPS group, on the other hand, recipients had significantly lower thrombocyte levels compared to donors, 168 $(130-216) \times 10^{9} / \mathrm{L}$ versus $206(126-274) \times 10^{9} / \mathrm{L}(p=0.037)$, respectively. However, no significant difference in the occurrence of thrombocytopenia between donors and recipients was found in the TAPS group. In addition, no significant differences in thrombocyte levels were found in the combined TTTS/TAPS group.

As shown in Table 3, early-onset sepsis occurred in $23.7 \%$ of the donors compared to $13.7 \%$ of the recipients $(p=0.049)$. Proven sepsis occurred in 4 neonates $(4.1 \%)$, all donor twins, and was due to Staphylococcus aureus in two neonates and coagulase-negative Staphylococcus in the other two neonates. Of the 7 donors with leukopenia, 4 (57\%) had early-onset neonatal sepsis, of which 1 had a positive blood culture (coagulase-negative Staphylococcus).

Risk factors for early-onset sepsis were maternal fever (8.1\%, 8/99), maternal GBS carrier $(2.0 \%, 2 / 99)$, prolonged rupture of membranes $(10.6 \%, 21 / 198)$, and maternal antibiotics during labor $(15.7 \%, 15 / 95)$.

We found no difference in the occurrence of NEC or neonatal mortality between donor and recipient twins. 


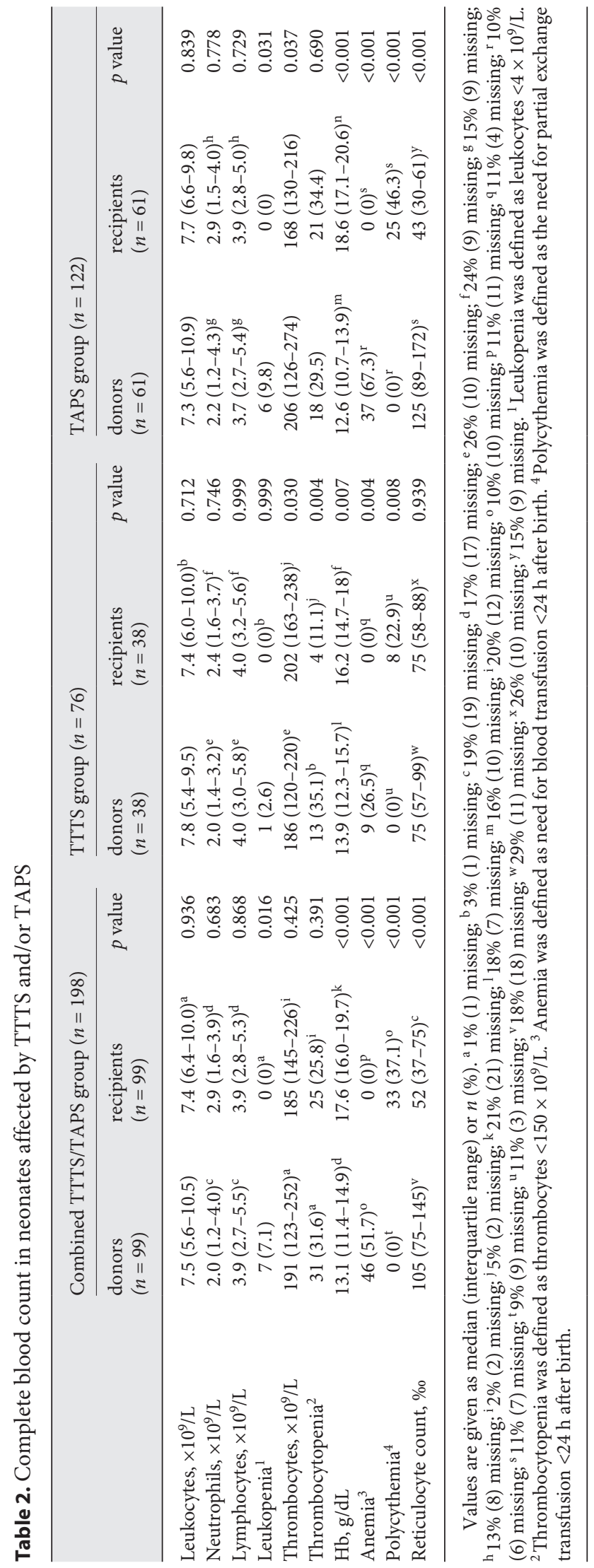

Table 3. Clinical outcome in neonates affected by TTTS or TAPS

\begin{tabular}{|c|c|c|c|}
\hline & \multicolumn{3}{|c|}{ Combined TTTS/TAPS group } \\
\hline & $\begin{array}{l}\text { donors } \\
(n=99)\end{array}$ & $\begin{array}{l}\text { recipients } \\
(n=99)\end{array}$ & $\begin{array}{l}p \\
\text { value }\end{array}$ \\
\hline \multicolumn{4}{|l|}{ Risk factors } \\
\hline Maternal fever & \multicolumn{3}{|c|}{$8(8.1)$} \\
\hline Maternal GBS carrier & \multicolumn{3}{|c|}{$2(2.0)$} \\
\hline Prolonged rupture of membranes & \multicolumn{3}{|c|}{$21(10.6)$} \\
\hline Maternal antibiotic use during & \multirow{2}{*}{\multicolumn{3}{|c|}{$15(15.7)$}} \\
\hline labor $^{\mathrm{a}}$ & & & \\
\hline Early-onset sepsis ${ }^{1}$ & $23(23.7)^{b}$ & $13(13.7)^{\mathrm{a}}$ & 0.049 \\
\hline Proven sepsis & $4(4.1)^{\mathrm{c}}$ & $0(0)^{\mathrm{d}}$ & 0.125 \\
\hline $\mathrm{NEC}^{2}$ & $4(4.1)^{\mathrm{b}}$ & $3(3.1)^{\mathrm{c}}$ & 0.999 \\
\hline Mortality & $10(10.1)$ & $6(6.1)$ & 0.388 \\
\hline
\end{tabular}

Values are given as $n$ (\%). ${ }^{\mathrm{a}} 4(4 \%)$ missing; ${ }^{\mathrm{b}} 2$ (2\%) missing; ${ }^{\mathrm{c}} 1$ (1\%) missing; ${ }^{\mathrm{d}} 3$ (3\%) missing. ${ }^{1}$ Sepsis was defined as the need for antibiotic therapy $<72 \mathrm{~h}$ postpartum and continuation of therapy because of clinical appearance, elevated C-reactive protein level, or positive blood culture. ${ }^{2}$ NEC was defined as Bell's stage $\geq 2$.

The incidence of NEC in donor and recipient twins was $4.1 \%(4 / 97)$ and $3.1 \%(3 / 95), p=0.999$, respectively. Neonatal mortality in donor and recipient twins was $10.1 \%$ $(10 / 99)$ versus $6.1 \%(6 / 99)(p=0.388)$, respectively. Use of full course antibiotics during admission was $25 \%(23 / 97)$ in donor and $14.4 \%(13 / 90)$ in recipient twins $(p=0.049)$.

\section{Discussion}

This is the first study analyzing the white blood cell line and leukocyte levels at birth in a large cohort of TTTS and TAPS twins. This study shows that the absolute leukocyte levels did not differ between donors and recipients. However, donor twins had significantly more often leukopenia (defined as a leukocyte count $<4 \times 10^{9} / \mathrm{L}$ ) compared to recipient twins. This increased risk of leukopenia was detected primarily in TAPS donor twins.

In TTTS and TAPS, almost all research on hematological complications involved investigations on the red blood cell line. Most studies show (as confirmed in this study) that donor twins have a significantly lower $\mathrm{Hb}$ level than recipients due to inter-twin transfusion through the placental vascular anastomoses.

The only report evaluating the occurrence of white blood cell abnormalities in TTTS was published in 1991 by Koenig et al. [5] in a small study in 5 twin pairs. The authors noted neutropenia (defined as below the lower limit of nor- 
mal for postnatal age, as established by Manroe et al. [10] in 1979) in all donors and in 4 out of 5 recipients. In addition, donor twins had considerably lower neutrophils than recipients and did not reach normal values for 4-8 days.

The responsible mechanism of reduced neutrophils in donors in the study by Koenig et al. [5] and the higher risk of leukopenia (primarily in TAPS donors) in our study is not known. We hypothesize that the occurrence of leukopenia could be related due to bone marrow repression as a result of increased erythropoiesis. As previously shown, and also confirmed in this study, TAPS donor twins have higher reticulocyte counts than recipients as a reaction to chronic anemia. Reticulocytosis and increased erythropoiesis was more prominent in TAPS donors than in TTTS donors, and this could explain the fact that leukopenia in this study was detected mainly in TAPS cases. The absence of reticulocytosis in the TTTS group could be due to the milder form of TTTS in this subgroup (mainly stages 1 and 2). Whether reticulocyte counts (and erythropoiesis) are increased in TTTS donors has not previously been reported in other cohorts.

We also hypothesized that leukopenia in TAPS donors could be related to intrauterine growth restriction. As previously reported, growth restriction in a fetus can lead to increased erythropoiesis due to chronic hypoxia, which in turn can suppress the production of other blood cell lines [11]. However, we found that the incidence of SGA was not more prominent in TAPS donors with leukopenia compared to those without leukopenia.

Another hypothesis for the higher risk of leukopenia in donors could be related to the inter-twin transfusion of white blood cells, alongside the inter-twin transfusion of red blood cells. However, this hypothesis is not supported by our results, since we found no differences in absolute levels of leukocytes between donors and recipients. Lastly, donors might have an accelerated consumption of leukocytes when compared to recipients, although the mechanism for this is not clear. In our study, we found a higher incidence of early-onset sepsis in donor twins, which might be related to or caused by leukopenia.

Although the mechanisms for an increased risk of leukopenia remain unclear, our results may have important clinical implications. As shown in our study, the risk of early-onset neonatal sepsis was higher in donors compared to recipients. Whether this risk of sepsis is related to the higher incidence of leukopenia or other possible confounding factors such as a higher risk of growth restriction in donors is not known. Nevertheless, leukopenia is a known risk factor for sepsis $[6,12]$, and clinicians should be aware of this discrepancy between donors and recipients.

Leukocyte Counts at Birth in TTTS and TAPS

Our results should be interpreted with care due to the retrospective design. Due to small cohorts of the TTTS and TAPS subgroups, the possibilities to perform subgroup analysis and corrections for confounders are limited and unreliable.

In addition, in the TTTS group, a selection bias towards milder TTTS cases was detected as almost half of the cases were affected by Quintero stage 1. Most severe TTTS cases in the Netherlands are primarily treated with laser surgery at our center and were excluded from this study when laser surgery was successful.

In conclusion, in TTTS and TAPS twins treated conservatively, donors seem to be at an increased risk for leukopenia and early-onset neonatal sepsis (especially in TAPS). Increased awareness of these infection risks at birth and routine measurements of leukocyte levels and infection parameters in donor twins are therefore advisable.

\section{Statement of Ethics}

The study protocol has been approved by the institute's committee on human research. No animal experiments were performed in this study.

\section{Disclosure Statement}

The authors have no conflicts of interest to declare. There was no sponsorship or funding arranged for this research.

References

Fetal Diagn Ther 2020;47:123-128 DOI: $10.1159 / 000500859$ 42.
1 Gucciardo L, Lewi L, Vaast P, Debska M, De Catte L, Van Mieghem T, et al. Twin anemia polycythemia sequence from a prenatal perspective. Prenat Diagn. 2010 May;30(5):438-

2 Verbeek L, Middeldorp JM, Hulzebos CV, Oepkes D, Walther FJ, Lopriore E. Hypoalbuminemia in donors with twin-twin transfusion syndrome. Fetal Diagn Ther. 2013;33(2): 98-102.

3 Verbeek L, Slaghekke F, Sueters M, Middeldorp JM, Klumper FJ, Haak MC, et al. Hematological disorders at birth in complicated monochorionic twins. Expert Rev Hematol. 2017 Jun;10(6):525-32.

4 Verbeek L, Slaghekke F, Hulzebos CV, Oepkes D, Walther FJ, Lopriore E. Hypoalbuminemia in donors with twin anemia-polycythemia sequence: a matched case-control study. Fetal Diagn Ther. 2013;33(4):241-5.

5 Koenig JM, Hunter DD, Christensen RD. Neutropenia in donor (anemic) twins involved in the twin-twin transfusion syndrome. J Perinatol. 1991 Dec;11(4):355-8. 
6 Christensen RD, Yoder BA, Baer VL, Snow GL, Butler A. Early-Onset Neutropenia in Small-for-Gestational-Age Infants. Pediatrics. 2015 Nov; 136(5):e1259-67.

7 Slaghekke F, Kist WJ, Oepkes D, Pasman SA, Middeldorp JM, Klumper FJ, et al. Twin anemia-polycythemia sequence: diagnostic criteria, classification, perinatal management and outcome. Fetal Diagn Ther. 2010;27(4):18190.
8 Senat MV, Deprest J, Boulvain M, Paupe A, Winer N, Ville Y. Endoscopic laser surgery versus serial amnioreduction for severe twinto-twin transfusion syndrome. $\mathrm{N}$ Engl J Med. 2004 Jul;351(2):136-44.

9 Bell MJ, Ternberg JL, Feigin RD, Keating JP, Marshall R, Barton L, et al. Neonatal necrotizing enterocolitis. Therapeutic decisions based upon clinical staging. Ann Surg. 1978 Jan;187(1):1-7.

10 Manroe BL, Weinberg AG, Rosenfeld CR, Browne R. The neonatal blood count in health and disease. I. Reference values for neutrophilic cells. J Pediatr. 1979 Jul;95(1):89-98.
11 Fustolo-Gunnink SF, Vlug RD, Smits-Wintjens VE, Heckman EJ, Te Pas AB, Fijnvandraat K, et al. Early-Onset Thrombocytopenia in Small-For-Gestational-Age Neonates: A Retrospective Cohort Study. PLoS One. 2016 May;11(5):e0154853.

12 Beaulieu E, Massé E, Dallaire F. Cord blood neutropenia is an independent predictor of early sepsis. J Perinatol. 2017 Nov; 37(11): $1204-9$. 